\title{
Comparative Evaluation of Academic Performance in Waikato Environment for Knowledge Analysis Using Multiple Classification Algorithms
}

\author{
${ }^{1}$ Asabe Sandra Ahmadu PhD, ${ }^{2}$ Etemi Joshua Garba PhD, ${ }^{3}$ Ally Dauda Ahmadu \\ ${ }^{1,2}$ Department of Computer Science, Modibbo Adama University Yola, Nigeria \\ ${ }^{3}$ ICT Centre, Federai University Wukari, Nigeria
}

\begin{abstract}
Data mining (DM) is the process of applying algorithms on large databases with the aim of discovering knowledge that would help in taking informed decisions by the management of academic institutions (Chalurapruek, $S$, et al, 2018). This paper seeks to discover the best classifiers to be used on educational data when using Waikato Environment for Knowledge Analysis (WEKA). The variables of importance namely carry-over, marital status, age range, entry mode and accommodation location were selected by $\mathbf{J 4 5}$ classifier. Four sampled datasets from four schools/faculties namely School of Physical Sciences (SPS), School of Environmental Studies (SES), School of Technology and Science Education (STSE), School of Agriculture and Agricultural Technology (SAAT) belonging to Modibbo Adama University of Technology (MAUTECH), Yola, Nigeria, were used for the DM task. All the classifiers available in WEKA suite were applied independently on the four different datasets. Notably, classifiers such as J48, NaiveBayes, Logistics and Regression gave better performances when compared with the rest. In the comparative analysis, the Regression model had the overall best performance of $98.366 \%, 99.3197 \%$, $\mathbf{9 6 . 3 9 6 4} \%$ and $96.875 \%$ on the four datasets respectively. The computed average performance of each of the four classifiers on the four datasets gave97.5446 \%, 94.6954\%, $\mathbf{9 5 . 6 7 0 1 2 5} \%$, and $97.739275 \%$ respectively.
\end{abstract}

Keywords: Multiple Classification Algorithms, Comparative Evaluation, Waikato Environment, Data mining.

\section{INTRODUCTION}

Data mining (DM) approaches have been originally used in business to predict items that are likely going to be purchased together or customers that will patronise new items marketed. DM is now applied in diverse fields notably amongst them newly emerging, is Educational Data Mining (EDM). Data can be collected form historical and operational data resident in the databases of educational institutes and can be personal or academic. In addition, it can be collected from e-learning systems, which have a large amount of information used by most institutes (Romero and Ventura 2007, Romero et al. 2008)

Data mining has several tasks such as association rule mining, classification and prediction, and clustering. Classification is one of the most useful techniques in data mining. There are several algorithms for data classification such as decision tree and Naïve Bayes classifiers (AlRadaideh and Nagi2012).

A lot of researches have been conducted using various classification techniques in order to improve performance and to handle various categories of data since research has shown that data is one of the factors that affect performance of an algorithm (Wittenand Frank2005).

One of the major challenges of using DM algorithm is that of choosing the right one to apply on a data set in order to get an optimistic performance. Luckily, WEKA has comprehensive algorithms that can be applied on the fly on a dataset or database so long as it is well cleansed without having to transform the data from one form to the other as the algorithms are varied. In this paper, we used the algorithms available in WEKA (as listed in Table 1) on our datasets and picked Naïve Bayes, Simple Logistics, Classification via Regression and J48 because they gave better performances. Consequently, in comparison with these four algorithms, Classification via Regression gave the best performance.

\section{LITERATURE REVIEW}

In this section, the classification techniques that have given optimistic performance on our datasets were reviewed. Also reviewed are some few works that are related to this article as employed by other researchers in order to find out the best classifier(s) on their datasets since research has proven that data plays a role in the performance of an algorithm.

\subsection{Data Mining}

Data Mining is the application of algorithms for extracting knowledge from large data or data warehouse. There are tools 
ISSN (online): 2581-3048

that help in either building the model or mining the data. In data mining, not a single technique is preferred over the other; a method is preferred depending on the kind of problem one wants to solve. Multiple methods can be integrated in order to solve a problem. Examples of these methods include Statistical, Decision Support Systems, Database Management and Warehousing, Machine Learning, Visualization etc. are all tools that interact and support a data-mining tool (Philip and Pedro, 1999).

\subsection{Classification}

Classification is the process of grouping data or concepts with similarities in a form of class of objects for the purpose of using it as a model on a similar data in order to accurately predict the unknown class of objects that exist in them (Kumar and Chadha 2011). The model is generated based on the analysis of a set of training data (that is, data objects for which the class labels are known) and is used to predict the classlabel of unclassified objects. One way of using WEKA is to apply a learning method to a dataset and analyze its output to extract information about the data.

Another is to apply several learners and compare their performances in order to choose one for prediction.

The learning methods are called classifiers (Witten and Fran, 2004). Classifiers in WEKA are the models for predicting nominal or numeric quantities. The learning schemes available in WEKA include Decision trees, Instancebased classifiers, Support vector machines, Multi-layer perceptions, logistic regression, and Bayes net.

In classification, the predicted variable can be either a binary or a categorical variable. Some popular classification methods in educational domains include decision trees, random forest, decision rules, step regression and logistic regression. Note that step regression and logistic regression, despite their names, are classifiers rather than regressors.

\subsection{WEKA}

WEKA is a data mining system that implements data mining algorithms. WEKA is a state-of-the-art facility for developing machine learning (ML) techniques and their application to real-world data mining problems. It is a collection of machine learning algorithms for data mining tasks. The algorithms can either be applied directly to a dataset or called from your own Java code. Weka contains tools for data pre-processing, classification, regression, clustering, association rules, and visualization (Michael and Gordon, 2004). Weka data mining tool can be used to mine a database directly instead of extracting data before mining as the case is obtained when using other machine learners/statistical tools.

Witten and Frank (2005) gave the breakdown of the associated functional names of classifiers available in WEKA as shown in Table 1.

Table 1: The Learning Schemes Available In WEKA

NAME FUNCTION

\begin{tabular}{ll}
\hline Bayes AODE & Averaged, one-dependence estimators \\
BayesNet & Learn Bayesian nets \\
\hline ComplementNaiveBayes & Build a Complement Naïve Bayes classifier \\
NaiveBayes & Standard probabilistic Naïve Bayes classifier \\
NaiveBayesMultinomial & Multinomial version of Naïve Bayes \\
NaiveBayesSimple & Implementation of Naïve Bayes \\
NaiveBayesUpdateable & Incremental Naïve Bayes classifier that learns one instance at a time \\
Trees ADTree & Build alternating decision trees \\
DecisionStump & Build one-level decision trees \\
Id3 & Basic divide-and-conquer decision tree algorithm \\
J48 & C4.5 decision tree learner (implements C4.5 revision 8) \\
LMT & Build logistic model trees \\
M5Rules & Obtain rules from model trees built using M5 \\
NBTree & Build a decision tree with Naïve Bayes classifiers at the leaves \\
RandomForest & Construct random forests \\
RandomTree & Construct a tree that considers a given number of random features at each node \\
REPTree & Fast tree learner that uses reduced-error pruning \\
UserClassifier & Allow users to build their own decision tree Rules \\
ConjunctiveRule & Simple conjunctive rule learner \\
DecisionTable & Build a simple decision table majority classifier \\
JRip & RIPPER algorithm for fast, effective rule induction \\
M5Rules & Obtain rules from model trees built using M5c \\
Nnge & Nearest-neighbour method of generating rules using non nested generalized exemplars \\
OneR & 1R classifier \\
Part & Obtain rules from partial decision trees built using J4.8 \\
\end{tabular}




$\begin{array}{ll}\begin{array}{l}\text { Prism } \\ \text { Ridor }\end{array} & \begin{array}{l}\text { Simple covering algorithm for rules } \\ \text { Ripple-down rule learner } \\ \text { ZeroR }\end{array} \\ \begin{array}{l}\text { PeastMedSq } \\ \text { PinearRegression }\end{array} & \begin{array}{l}\text { Robust regression using the median rather than the mean } \\ \text { Standard linear regression }\end{array} \\ \begin{array}{l}\text { Logistic } \\ \text { MultilayerPerceptron }\end{array} & \begin{array}{l}\text { Build linear logistic regression models } \\ \text { Backpropagation neural network }\end{array} \\ \text { PaceRegression } & \text { Build linear regression models using Pace regression } \\ \text { RBFNetwork } & \text { Implements a radial basis function network } \\ \text { SimpleLinearRegression } & \begin{array}{l}\text { Learn a linear regression model based on a single attribute } \\ \text { Build linear logistic regression models with built-in attribute selection }\end{array} \\ \text { SimpleLogistic } & \text { Sequential minimal optimization algorithm for support vector classification } \\ \text { SMO } & \text { Sequention }\end{array}$

J48

$\mathrm{J} 48$ is WEKA version of C4.5 decision tree algorithm. It permits decision makers to change confidence threshold responsible for tree pruning to produce minimum required number of instances permitted at a leaf (Aftarczuk, 2007). The C4.5 algorithmis an extension of ID3 algorithms both developed by Ross Quinlan in 1989 and 1993 with additional features to handle missing values, classification of continuous attributes, tree pruning and rule derivation. Decision trees represent rules; rules can readily be expressed in English so that humans can understand them. They are produced by algorithms that identify various ways of splitting a dataset into branch-like segments. Decision tree algorithm is a popular procedure today because of its ease of implementation and in particular because of the possibility for the results to be graphically displayed (Osmanbegović \& Suljić, 2012).

\subsubsection{Naive Bayes}

The Bayesian Classification represents a supervised learning method as well as a statistical method for classification of both discrete and Continuous variables. Assumes an underlying probabilistic model and it allows us to capture uncertainty about the model in a principled way by determining probabilities of the outcomes. It can solve diagnostic and predictive problems. This Classification is named after Thomas Bayes (1702-1761), who proposed the Bayes Theorem (software.ucv.ro).

According touros@krcadinac.com (2015) Naive Bayes makes two "naïve" assumptions over attributes:

i. All attributes are a priori equally important;

ii. All attributes are statistically independent (value of one attribute is not related to a value of another attribute).

These assumptions mostly are not true, but in practice, the algorithm gives good results

$\mathrm{P}(\mathrm{H} \backslash \mathrm{E})=\frac{P(E \backslash H) * P(H)}{P(E)}$

Where
$\mathrm{H}$ - Hypothesis

E - Evidence related to the hypothesis H, i.e., the data to be used for validating (accepting/rejecting) the hypothesis $\mathrm{H}$

$\mathrm{P}(\mathrm{H})$ - probability of the hypothesis (prior probability)

P (E) - probability of the evidence i.e., the state of the world described by the gathered data

$\mathrm{P}(\mathrm{E} \mid \mathrm{H})$ - (conditional) probability of evidence $\mathrm{E}$ given that the hypothesis $\mathrm{H}$ holds

$\mathrm{P}(\mathrm{H} \mid \mathrm{E})$ - (conditional) probability of the hypothesis $\mathrm{H}$ given the evidence $\mathrm{E}$

Naive Bayes classification can be use in:

i) Naive Bayes text classification

ii) Spam filtering (a mechanism that distinguishes illegitimate spam email from legitimate email).

iii) Recommender Systems (for filtering unseen information and predicting whether a user would like a given resource).

\subsubsection{Regression}

Regression is used to evaluate the relative impact of a predictor variable on a particular outcome. However, regression fits functional dependencies between factors more. The term functional dependency refers to a factor being a partial determinant of an occurrence. For example, the availability of Internet facility in an institution is a determinant factor for performance. There are other types of regression e.g. multiple linear, logistic, and ordinal (Zou et al. 2003). The most popular regression within educational data mining is linear regression. Regressions such as Neural Networks and Support Vector Machines, which are prominent in other data mining domains. They are somewhat less common in educational data mining. This is thought to be because the high degrees of noise and multiple explanatory factors in educational domains often lead to conservative versions being more successful. Regressions can be validated using the same overall techniques as that in classifiers, often using the metrics of linear correlation or root mean squared error (RMSE). 
ISSN (online): 2581-3048

\subsubsection{Simple Logistic}

Simple Logistic builds logistic regression models fitting them using Log it Boost with simple regression functions as base learners and determining how many iterations to perform using cross-validation which supports automatic attribute selection (Landwehr et al. 2003). Logisticis an alternative implementation for building and using a multinomial logistic regression model with a ridge estimator to guard against over fitting by penalizing large coefficients (Cessie and Houwelingen, 1992).

\subsubsection{Evaluation}

Evaluation means to assess or appraise the performance of instances in a data set or to judge something with respect to its worth or significance or quality. The predictive performance of an algorithm can be evaluated using statistical formula or an automated system (Machine Learning tool) which outputs statistical results summarizing how accurately the classifier was able to predict the true class of the instances under the chosen test module. The set of measurements is derived from the training data (Witten and Frank 2005).

\subsection{Related Works}

Data mining techniques can be used in educational field to enhance our understanding of learning process to focus on identifying, extracting and evaluating variables related to the learning process of students (El-Halees 2009).

Kotsiantis, et al. (2004) applied five classification algorithms namely Decision Trees, Perception-based Learning, Bayesian Nets, Instance-Based Learning and Rulelearning to predict the performance of Computer Science students. The filtration was based on variable selection technique that was used to select highly influencing variables; and all the above five classification models were constructed. It was noticed that the NaïveBayes yielded high predictive accuracy (74\%) for two-class (pass/fail) dataset.

Sudha and Kumaravel (2017) conducted a comparative analysis between two data mining tools ROSE2 (which uses Rough set theory base classification method) and Tanagra tool which has the following four algorithms inbuilt: NaïveBayes, C4.5, C-RT, and K-NN. Experiment was performed in order to predict the disease in patients using instances involved in arrhythmia disease. The accuracy level showed that Tanagra performed better than Rose2 tool, but comparatively, C4.5 superseded the other four algorithms with prediction Accuracy of $85 \%$ compared to NaïveBayes, C-RT, K-NN and ROSE2 which had $75.71 \%, 74 \%, 72 \%$, and $70.25 \%$ respectively.
Yadav and Pal (2012) conducted a research on frequently used decision tree classifiers C4.5, ID3 and CART so as to discover the best classifier for the prediction of student's performance in First Year of engineering examinations. The decision trees predicted the number of students who are likely to pass, fail or promoted to next year. A comparative analysis of the results showed that $\mathrm{C} 4.5$ technique had the highest accuracy of $67.78 \%$ - which is acceptable when compared to ID3 and CART algorithms.

Aziz et al. (2014) developed Students' Academic Performance prediction models for the first semester students of the department of Computer Science Universiti Sultan Zainal Abidin (UniSZA) using three selected classification methods; Naïve Bayes, Rule Based, and Decision Tree. The comparative analysis conducted discovered that the best classification model, which showed the best result, is that developed using Rule Based and Decision Tree algorithm compared to the model developed from the Naïve Bayes algorithm.

\section{METHODOLOGY}

\section{Discuss: Theories, techniques and tools used in this paper}

The data-mining task used sampled datasets collected from four schools: SPS, SES, STSE and SAAT. The four different datasets were used in the comparative analysis of the classifiers J48, NaiveBayes, Logistics, and Regression. Consequently, 10-fold cross-validations were used on each of the classifiers trained on each of the datasets so that minority representations would not be overlooked.

\section{EXPERIMENTS}

\subsection{Performance}

The word performance is used in two scenarios: a) Performance is known as the percentage proportion of all classified cases in the dataset (the variable that assesses the efficiency of a model during analysis); b) It was used in naming one of the attributes used in the dataset as detailed in Table 2.

Table 2: CGPA classified into two categories

\begin{tabular}{|c|c|}
\hline CGPA & PERFORMANCE \\
\hline Above 2.39 & Yes \\
\hline Below 2.40 & No \\
\hline
\end{tabular}

Predictive accuracy (Performance)

$$
=\frac{T P+T N}{T P+T N+F P+F N} 100 \%
$$


ISSN (online): 2581-3048

Table 3: Confusion matrix

\begin{tabular}{|l|l|l|}
\cline { 2 - 3 } \multicolumn{1}{c|}{} & Predicted positive & Predicted negative \\
\hline Actual positive & TP & FN \\
\hline Actual negative & FP & TN \\
\hline
\end{tabular}

\subsection{Confusion Matrix}

The four classifiers: J48, NaiveBayes, Logistics and Regression were ran on the four different datasets of SPS, SES, STSE and SAAT; and their performances and confusion matrix have been summarised in Tables 4 to 7 .

Table 4: Confusion Matrix for J48 Classifier on the Four selected datasets

\begin{tabular}{|l|l|l|l|}
\hline & Yes & No & \multicolumn{1}{|c|}{ SPS } \\
\hline Yes & 44 & 4 & Prediction Accuracy \\
\hline No & 0 & 258 & $98.6928 \%$ \\
\hline & Yes & No & SES \\
\hline Yes & 44 & 4 & Prpdiction Accuracy \\
\hline No & 0 & 258 & $98.6928 \%$ \\
\hline Yes & 91 & 2 & Prediction Accuracy \\
\hline No & 2 & 16 & $96.3964 \%$ \\
\hline Yes & No & SAAT \\
\hline Yes & 91 & 2 & Prediction Accuracy \\
\hline Yes & 2 & 16 & $96.3964 \%$ \\
\hline
\end{tabular}

Table 5: Confusion Matrix for NaiveBayes Classifier on the Four selected datasets

\begin{tabular}{|l|l|l|l|}
\hline & Yes & No & \multicolumn{1}{|l|}{ SPS } \\
\hline Yes & 44 & 4 & Prediction Accuracy \\
\hline No & 0 & 258 & $98.6928 \%$ \\
\hline Yes & Yes & No & SES \\
\hline No & 207 & 0 & Prediction Accuracy \\
\hline Yes & 92 & 84 & $99.3174 \%$ \\
\hline No & 3 & 15 & STSE \\
\hline Yes & No & Prediction Accuracy \\
\hline Yes & 23 & 2 & Prediction Accuracy \\
\hline No & 3 & 4 & $84.375 \%$ \\
\hline
\end{tabular}

https://doi.org/10.47001/IRJIET/2021.507013

Table 6: Confusion Matrix for Logistic on the Four selected datasets

\begin{tabular}{|c|c|c|c|}
\hline & Yes & No & SPS \\
\hline Yes & 38 & 10 & Prediction Accuracy \\
\hline \multirow[t]{2}{*}{ No } & 8 & 250 & $94.1176 \%$ \\
\hline & Yes & No & SES \\
\hline Yes & 207 & 0 & Prediction Accuracy \\
\hline \multirow[t]{2}{*}{ No } & 2 & 84 & $99.3174 \%$ \\
\hline & Yes & No & STSE \\
\hline Yes & 92 & 1 & Prediction Accuracy \\
\hline \multirow[t]{2}{*}{ No } & 4 & 14 & $95.4955 \%$ \\
\hline & Yes & No & SAAT \\
\hline Yes & 25 & 0 & Prediction Accuracy \\
\hline No & 2 & 5 & $93.75 \%$ \\
\hline
\end{tabular}

Table 7: Confusion Matrix for Regression on the Four selected datasets

\begin{tabular}{|c|c|c|c|}
\hline & Yes & No & SPS \\
\hline Yes & 43 & 5 & Prediction Accuracy \\
\hline \multirow[t]{2}{*}{ No } & 0 & 258 & $98.366 \%$ \\
\hline & Yes & No & SES \\
\hline Yes & 207 & 0 & Prediction Accuracy \\
\hline \multirow[t]{2}{*}{ No } & 2 & 85 & $99.3197 \%$ \\
\hline & Yes & No & STSE \\
\hline Yes & 91 & 2 & Prediction Accuracy \\
\hline \multirow[t]{2}{*}{ No } & 2 & 16 & $96.3964 \%$ \\
\hline & Yes & No & SAAT \\
\hline Yes & 25 & 0 & Prediction Accuracy \\
\hline No & 1 & 6 & $96.875 \%$ \\
\hline
\end{tabular}


4.3 Performance of J48, NaiveBayes, Simple Logistic and Regression Classifiers Tabulated

Table 8: Performance of the selected best four models in WEKA

\begin{tabular}{|l|c|c|c|c|c|}
\hline & \multicolumn{5}{|c|}{ PERFORMANCE IN \% } \\
\hline MODELS & SPS & SES & STSE & SAAT & AVERAGE \\
\hline J48 & 98.69 & 98.69 & 96.40 & 96.40 & 97.55 \\
\hline NaiveBayes & 98.69 & 99.32 & 96.40 & 84.38 & 94.70 \\
\hline Simple Logistic & 94.12 & 99.32 & 95.50 & 93.75 & 95.67 \\
\hline Regression & 98.37 & 99.32 & 96.40 & 96.88 & 97.74 \\
\hline
\end{tabular}

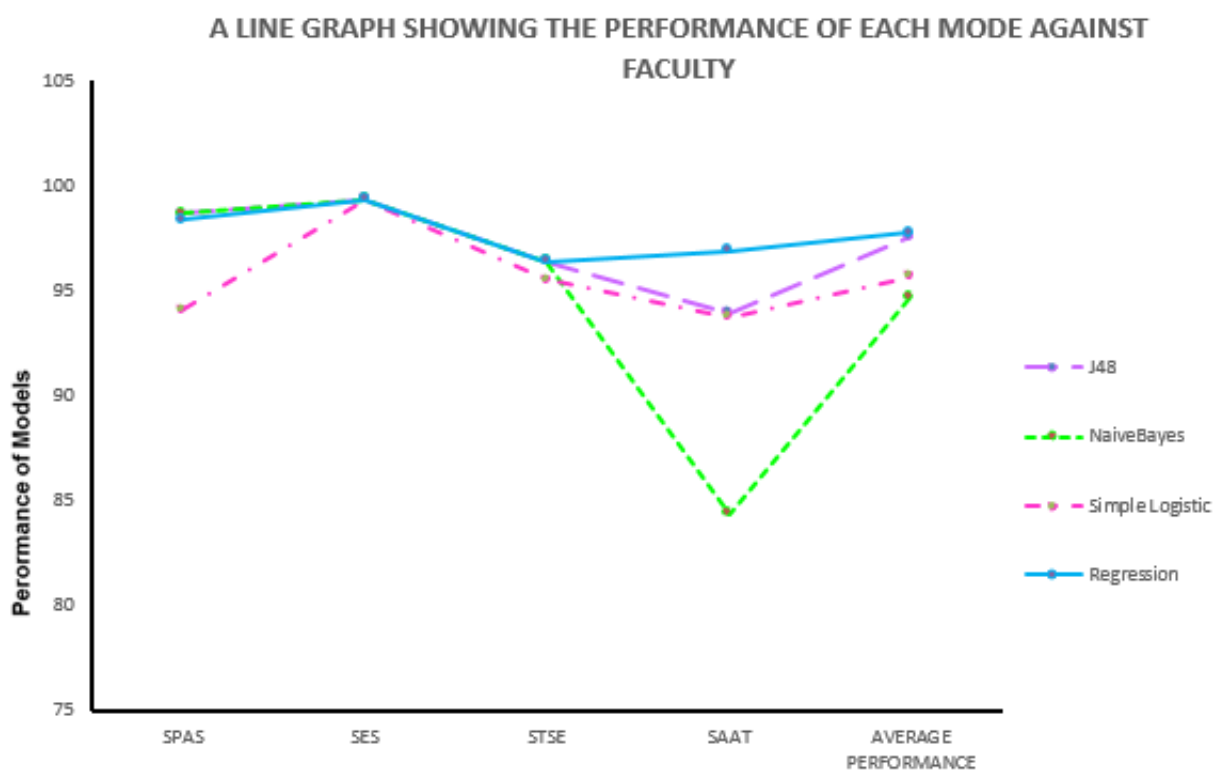

Figure 1: Performance of four selected sampled Classifier in WEKA

\section{DISCUSSION}

J48 algorithm was applied on the sampled data seven hundred and seventy-three (743) records put together collected from four Schools/Faculties of SPS, SES, STSE and SAAT in MAUTECH, Yola Nigeria for the selection of variables of importance that were subsequently used on the rest of classifiers. The attributes carry-over, marital status, age range, entry mode, and accommodation location were picked as the variables of importance.

We then tried the different classifiers in WEKA suite on the four data sets independently. The classifiers J48, NaiveBayes, Simple Logistics and Regression gave better performances with Regression giving the best performance of 98.366, 99.3197, 96.3964 and 96.875 respectively. The average performance of each classifier was computed on the four datasets and the results obtained were 97.5446, 94.6954, 95.670125 and 97.739275 respectively.
The performances of these selected algorithms were plotted in a line graph as depicted in Figure1. The results for J48, NaiveBayes, Simple Logistics and Regression are almost the same for the Schools/Faculties SES and STSE. Since we have tried all classifiers on varied datasets, the results obtained gave varied performances which imply that data also plays an important role in the performance of an algorithm.

\section{CONCLUSION}

The comparative analysis of results from the four different classifiers J48, Logistics, Regression and NaiveBayes revealed that Regression model had the overall best performance for the four sampled schools of SPS, SES, STSE and SAAT. From the experimental results obtained, we recommend that Regression, J48, Simple Logistic and NaiveBayesto be used on EDM when using WEKA suite. Note that the listing of their classifiers is in order of preference. We also draw conclusion that the success of a data-mining task is dependent on the algorithm and data used. 
ISSN (online): 2581-3048

\section{REFERENCES}

[1] Aftarczuk, K. (2007). Evaluation of selected data mining algorithms implemented in Medical Decision Support Systems. Blekinge: Blekinge Institute of Technology School of Engineering.

[2] Al-Radaideh, Q. A. and Nagi, E. A. (2012) Using Data Mining Techniques to Build a Classification Model for Predicting Employees Performance (IJACSA) International Journal of Advanced Computer Science and Applications, Vol. 3, No. 2, 2012. www.ijacsa.thesai.org

[3] Aziz, A. A., Ismail, N. H., \& Ahmad, F. (2014). Proceeding of the International Conference on Artificial Intelligence and Computer Science (AICS 2014), Bandung, INDONESIA. (e-ISBN978-96711768-8-7). Organized by http://WorldConferences.net Retrieved on 15/03/2016.

[4] Cessie, S.L., and Houwelingen J.C.V.(1992) Ridge Estimators in Logistic Regression University of Leiden, Neitherlands. 1992 Royal statistical society. 41, No.1 pp

191-201, www.inf.unibz.it/dis/teaching/DWDM/project2010, Retrieved on 07/09/2017.

[5] El-Halees, A (2009) "Mining Students Data to Analyze E-Learning Behavior: A Case Study".

[6] Kotsiantis, S., Pierrakeas, C. and Pintelas, P. (2004). Prediction of Student's Performance in Distance Learning Using Machine Learning Techniques\|, Applied Artificial Intelligence, 18(5) 411-426.

[7] Kumar, V. And Chadha, A. ( 2011). An Empirical Study of the Applications of Data Mining Techniques in Higher Education (IJACSA) International Journal of Advanced Computer Science and Applications, http://ijacsa.thesai.org Retrieved: 10/06/2014.

[8] Landwehr, N. (3003) Logistic Model trees www.cs. waikato.ac.nz/ml/publications/2003/landwehretal.ps

[9] Michael J. A. B. and Gordon S. L. (2004), Data Mining Techniques, 2nd ed., Wiley Publishing Inc., USA, www.cs.waikato.ac.nz/ml/weka Retrieved on 15/03/2016.

[10] Osmanbegović, E., \& Suljić, M. (2012). Data Mining Approach For Predicting Student Performance. Economic Review - Journal of Economics and Business, X(1), 3-12.

[11] Pal, A. K and Pal, S. (2013) Classification Model of Prediction for Placement of Students, I.J.Modern Education and Computer Science, pp 49-56 Published Online November 2013 in MECS http://www.mecspress.org Retrieved on 22/09/2017.

[12] Philip, C. and Pedro, M. (1999). On the use of support vector machines for phonetic classification. Acoustics,
Speech, and Signal Processing. 1999 IEEE International Conference. (2).

[13] Romero, C. and Ventura, S. (2007) 'Educational data Mining: A Survey from 1995 to 2005', Expert Systems with Applications (33), pp. 135-146.

[14] Romero, C. , Ventura, S. and Garcia, E. (2008) 'Data mining in course management systems: Moodle case study and tutorial', Computers \& Education, vol. 51, no. $1, \quad$ pp. 368-384. Software.ucv.ro/ cmihaescu/ro/teaching/AIR/docs/Lab 4-NaiveBaye Retrieved on 07/09/2017.

[15] Sudha, M. And Kumaravel, A. (2017) Comparative Analysis between Rough set theory and Data mining algorithms on their prediction. Global Journal of Pure and Applied Mathematics. ISSN 0973-1768 Volume 13, Number 7 (2017), pp. 3249-3260 @ Research India Publications http://www.ripublication.com Retrieved: 11/09/2017.

[16] uros@krcadinac.com. URL: http://krcadinac.com... (2015) Naive Bayes classifier. ai.fon.bg.ac.rs/wpcontent/uploads/2015/04/Classification-Naive-Bayes2015.pdf Retrieved on 07/09/2017.

[17] Witten, I. H. And Frank, E. (2005); "Data Mining Practical Machine Learning Tools and Techniques", Second Edition, Morgan Kaufmann Publishers is an imprint of Elsevier.500 Sansome Street, Suite 400 San Francisco, CA 94111. pp.267-pp320, www.cs.waikato.ac.nz/ml/weka Retrieved on 15/03/2016.

[18] Yadav, S. K. And Pal, S. (2012) Data Mining: A Prediction for Performance Improvement of Engineering Students using Classification. World of Computer Science and Information Technology Journal (WCSIT) ISSN: 2221-0741 Vol. 2, No. 2, 51-56, 2012, https://www.researchgate Retrieved on 15/03/2016.

[19] Zou, K. H., Tuncali, K., and Silverman, S. G. (2003) Statistical Concepts Series in Radiology, Published online 10.1148/radiol.2273011499 Radiology 2003; 227:617-628 Vol.(3) pp 617-622, https://www.coursehero Retrieved on 07/09/2017.

[20] Chalurapruek S, Dee T. S., Johari R., Kizilcec R. F and Steven M. L (2018), How a Data Driven Course Planning Tool Affects college students GPA, Evidence from two field experiments: In Proceedings o Fifth Annual ACM Conference on Learning at Scale, Association $\mathrm{O}$ Computing Machineries, https://doi.org/10.1145/3231644.3231668 Google Scholar.

[21] Naïve Bayes Classifier is an approach that adopts the Bayes theorem, by combining previous knowledge with new knowledge.iJES - Vol. 7, No. 2, 2019. 
ISSN (online): 2581-3048

[22] The Naive Bayes algorithm is simple probabilistic classification. This algorithm calculates a set of probabilities by calculating the frequency and combination of values in a particular data set (Patil, T. R. (2013) "Performance Analysis of Naive Bayes and
https://doi.org/10.47001/IRJIET/2021.507013

J48 Classification Algorithm for Data Classification," Int. J. Comput. Sci. Appl. ISSN 0974-1011, vol. 6, no. 2, pp. $256-261)$.

\section{Citation of this Article:}

Asabe Sandra Ahmadu PhD, Etemi Joshua Garba PhD, Ally Dauda Ahmadu, "Comparative Evaluation of Academic Performance in Waikato Environment for Knowledge Analysis Using Multiple Classification Algorithms" Published in International Research Journal of Innovations in Engineering and Technology - IRJIET, Volume 5, Issue 7, pp 73-80, July 2021. Article DOI https://doi.org/10.47001/IRJIET/2021.507013 